\title{
IFUP-TH/2013-11 \\ Vortex lattices in three-component Bose-Einstein condensates under rotation: simulating colorful vortex lattices in a color superconductor
}

\author{
Mattia Cipriani ${ }^{1}$, Muneto Nitta ${ }^{2}$ \\ ${ }^{1}$ University of Pisa, Department of Physics "E. Fermi", INFN, Largo Bruno Pontecorvo 7, 56127, Italy \\ ${ }^{2}$ Department of Physics, and Research and Education Center for Natural Sciences, \\ Keio University, 4-1-1 Hiyoshi, Yokohama, Kanagawa 223-8521, Japan
}

\begin{abstract}
We study vortex lattices in three-component BECs under rotation, where three kinds of fractional vortices winding one of three components are present. Unlike the cases of two-component BECs where the phases of square and triangular lattices are present depending on the intercomponent coupling constant and the rotation speed, we find triangular ordered "colorful" vortex lattices where three kinds of fractional vortices are placed in order without defects, in all parameter regions where the inter-component coupling $g^{\prime}$ is less than the intra-component coupling $g$. When $g>g^{\prime}$ on the other hand, we find the phase separation: In a region where one component is present, the other two components must vanish, where we find ghost vortices in these two components whose positions are separated. In the boundary $g=g^{\prime}$, the accidental $U(3)$ symmetry is present, in which case two vortices in different components are close to each other in some regions.
\end{abstract}

\section{INTRODUCTION}

One of the growing topics in condensed matter physics, high energy physics, and astrophysics are multicomponent condensations. In this sense, interesting fields of research are exotic superconductors, multi-component or spinor Bose-Einstein condensates (BECs) of ultra-cold atomic gases, superfluid ${ }^{3} \mathrm{He}$, exciton-polariton condensates, nonlinear optics, hadronic matter composed of neutron and proton Cooper pairs, and quark matter composed of di-quark condensates consisting of quark Cooper pairs. In these systems, fractional vortices can be created by rotating superfluids or BECs or by applying magnetic field on superconductors. Fractional vortices are characterized by rational or fractional quantized circulations for superfluids or BECs and fluxes for superconductors, as found in various systems: superfluid ${ }^{3} \mathrm{He}$ [1, 2], $p$-wave superconductors [1, 3] [5], multigap superconductors [69], spinor BECs [10, 11], multi-component BECs [12 24, exciton-polariton condensates [25, 26], nonlinear optics [27], and color superconductors as quark matter [28 34].

Theoretical and experimental investigations of the properties of vortices are easily done in BECs of ultracold atomic gases [35], which reveal to be the ideal physical setting among the others for this purpose. In the theory side, BECs can be quantitatively well described in the mean-field theory by using the Gross-Pitaevskii (GP) equation. Experimentally, BECs are quite flexible and controllable systems being the atomic interaction tunable through a Feshbach resonance [36. In addition, the condensates can be visualized directly by means of optical techniques. Thus far, two-component BECs have been realized by using the mixture of atoms with two hyperfine states of ${ }^{87} \mathrm{Rb}$ [37] or the mixture of two different species of atoms [38 40]. In Refs. [13 19], the phase diagram of the vortex lattice forming in two-component BECs was studied, and a rich variety of lattices was found. When the inter-component coupling $g^{\prime}$ is increased, different configurations from an Abrikosov's triangular lattice of fractional vortices to a square lattice of fractional vortices have been obtained [13 15] when $g^{\prime}<g$.

On the other hand, particularly interesting cases in high energy physics and astrophysics include quark matter composed of di-quark condensates consisting of quark Cooper pairs [41, which may be realized in the core of neutron stars. When up, down, strange quarks participate in forming Cooper pairs, the order parameter $\Delta$ becomes a $3 \times 3$ matrix, on which color $S U(3)$ and flavor $S U(3)$ symmetries act from left and right, respectively. The Ginzburg-Landau description is available in the perturbation theory of quantum chromo dynamics. In the ground state, for which the condensate can be considered to be in a diagonal form in color and flavor without loss of generality, the color and flavor symmetries are spontaneously broken down into a diagonal combination of them. For this reason, this phase is called the color-flavor locked phase. Since color symmetry is spontaneously broken and completely screened, quark matter in this phase is a color superconductor. At the same time, it is a superfluid since the baryon $U(1)$ symmetry is spontaneously broken. Consequently, vortices appearing in this phase carry both the quantized circulations and quantized color magnetic fluxes 28 31. They are referred to as non-Abelian since the color fluxes they carry are non-Abelian magnetic fields. The circulation which they carry is quantized to be $1 / 3$ of the unit circulation, due to three components having non-zero value in the ground state. The interaction between non-Abelian vortices was calculated in [29] to be $1 / 3$ of that between integer quantized superfluid vortices, which implies the formation of a colorful vortex lattice when the color superconductor is rotating as in conventional superfluids. The phenomenon for which the vortex lattice behaves as a polarizer when light passes through it [32] is one of the interesting consequences of the aforementioned properties of quark matter in the color-flavor locked phase. Another interesting con- 
sequence is the existence of a colorful boojum [34] at the interface between a color superconductor and the confining phase in which all color degrees of freedom must be canceled out. In the latter, three color magnetic fluxes, say red, blue, and green, with $1 / 3$ circulations are combined together to a purely superfluid colorless vortex with the unit circulation, which can penetrate into the confining phase. Even though the colorful vortex lattice is one of the most important signals of whether the color-flavor locked phase is realized or not in the core of neutron stars rotating very rapidly, there have been no quantitative studies of colorful vortex lattices thus far, because of large degrees of freedom of non-Abelian $S U(3)$ gauge fields.

The purpose of this paper is to propose simulating certain aspects of colorful vortex lattices in a rotating color superconductor by using a BEC of ultra-cold atomic gases. We create $1 / 3$ quantized vortices with three different "colors" by preparing a rotating three-component BEC. Vortex lattices in three-components BEC have been investigated in the context of $\mathrm{F}=1$ spinor $\mathrm{BEC}$ [2]; we propose a non-spinor system for this analysis. Because our purpose is to simulate a color superconductor, we consider the symmetric case where all three intracomponent (inter-component) couplings are the same, $g_{11}=g_{22}=g_{33} \equiv g\left(g_{12}=g_{23}=g_{31} \equiv g^{\prime}\right)$, and all chemical potentials and masses are the same as the case of the mixture of atoms with different hyperfine states. Combining a set of three different vortices together results in an integer vortex without a "color." These features are the same with $1 / 3$ quantized vortices in the color superconductor. We solve the GP equation for a rotating three-component BEC subject to a harmonic trapping potential by an imaginary time propagation, and find "ordered" Abrikosov's triangular lattices in all parameter ranges of $g^{\prime}<g$. Here, the term "ordered" implies that each of the three different fractional vortices winding around the three different components constitutes an Abrikosov's triangular lattice without defects, and that the total configuration is also an Abrikosov's triangular lattice. In other words, we did not find any defects or displacements of vortices with different colors. This situation is in contrast to the cases of two-component BECs [13 19] in which there exist an Abrikosov's triangular lattice and a square lattice of fractional vortices in $g^{\prime}<g$, depending on the inter-component coupling $g^{\prime}$ as denoted above. When $g^{\prime}=g$ the zeros of the density of the different components can be overlapped. However, the comparison between plots of the phase of the order parameter of each component shows that the vortices are not coincident. In the parameter range $g<g^{\prime}$ of the phase separation, we find that vortex sheets are present like the case of two component BECs [21]. In a region where one component is present, the other two components are absent because of the phase separation. In that region, ghost vortices of these two components are present and they are separated, even if there is no symmetric ordering as found for $g^{\prime}<g$.

\section{GROSS-PITAEVSKII ENERGY FUNCTIONAL}

The energy functional of the Gross-Pitaevskii equations for the rotating BECs subject to a trapping harmonic potential can be written as:

$$
\begin{aligned}
E= & \int d^{3} r\left\{\sum _ { i = 1 , 2 , 3 } \Psi _ { i } ^ { * } \left[\frac{1}{2}\left(\frac{1}{i} \nabla-\Omega \hat{\boldsymbol{z}} \times \boldsymbol{r}\right)^{2}\right.\right. \\
& \left.\quad+\frac{r^{2}}{2}\left(1-\Omega^{2}\right)-\mu_{i}\right] \Psi_{i} \\
& +\frac{1}{2} g_{1}\left|\Psi_{1}\right|^{4}+\frac{1}{2} g_{2}\left|\Psi_{2}\right|^{4}+\frac{1}{2} g_{3}\left|\Psi_{4}\right|^{4} \\
& \left.+g_{12}\left|\Psi_{1}\right|^{2}\left|\Psi_{2}\right|^{2}+g_{23}\left|\Psi_{2}\right|^{2}\left|\Psi_{3}\right|^{2}+g_{31}\left|\Psi_{3}\right|^{2}\left|\Psi_{1}\right|^{2}\right\}
\end{aligned}
$$

where the derivatives include $(r, \theta)$ coordinates. We measure distances and energies in terms of $b=\sqrt{\hbar / m \omega}$ and $\hbar \omega$ respectively, where $m$ is the mass of the atoms and $\omega$ is the frequency of the trapping harmonic potential. As denoted in the introduction, we consider the symmetric case,

$$
\begin{gathered}
g_{11}=g_{22}=g_{33} \equiv g, \quad g_{12}=g_{23}=g_{31} \equiv g^{\prime} \\
m_{1}=m_{2}=m_{3} \equiv m, \quad \mu_{1}=\mu_{2}=\mu_{3} \equiv \mu
\end{gathered}
$$

When $g \neq g^{\prime}$, the system is $S_{3}$ symmetric under exchanges $\Psi_{i} \leftrightarrow \Psi_{j}$. When $g=g^{\prime}$, the system is $U(3)$ symmetric $\Psi_{i} \rightarrow \Psi_{i}^{\prime}=U_{i}{ }^{j} \Psi_{j}$ with $U \in U(3)$.

\section{VORTEX LATTICES}

We minimize the free energy (1) by the non-linear conjugate gradient method (the imaginary time propagation) in the FREEFEM ++ package. In all the numerical simulations we take $g=1, \Omega=0.96$ and $\mu=6$. We calculate the ground state of the system by minimizing the energy functional (1).

We first start as the initial configuration from the ground state of a non-rotating BEC where no vortices are present. We fix the rotation speed and let the vortices form in response to the rotation. We find that the vortices are nucleated as integer vortices disposed in an Abrikosov lattice for each value of $g^{\prime}$. This implies that an integer vortex composed of three different fractional vortices, $\Psi_{i} \sim e^{i \theta}$ with the other two having no winding, is metastable. We show some of the results we obtained in Fig. 1. The defect in the lattice of Fig. 1 (c) appears by chance and it is metastable.

Next, we introduce a perturbation in the lattice of integer vortices by splitting the one near the center of the cloud into a set of three different fractional vortices, with very small spacing. We use this perturbed configuration as the initial configuration in the next step and then obtain the final configuration for each value of $g^{\prime}$. We can 
(a) $\delta=0.5$

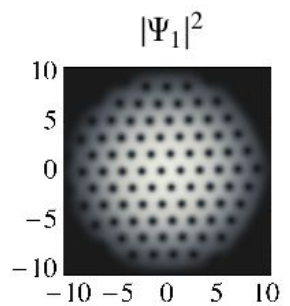

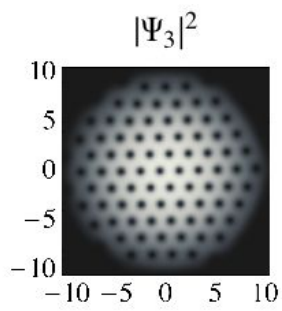

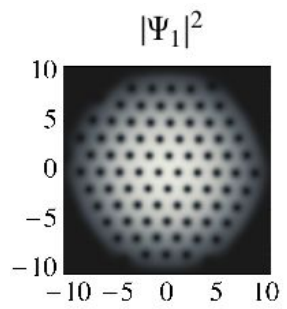

(b) $\delta=0.9$
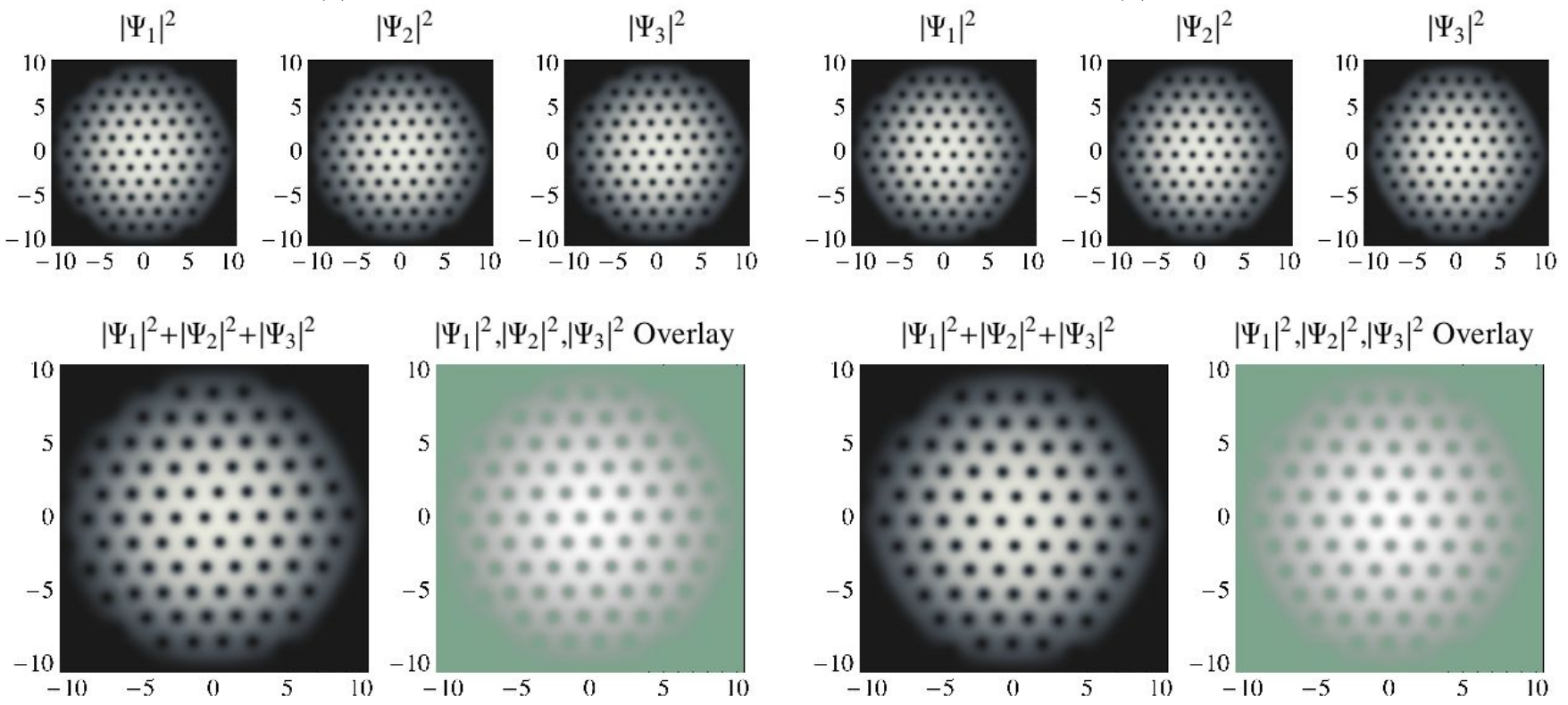

(c) $\delta=1$
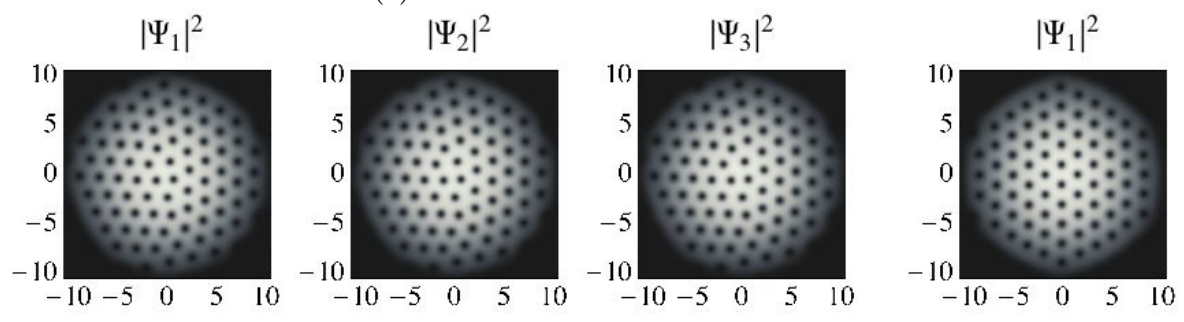

(d) $\delta=1.5$
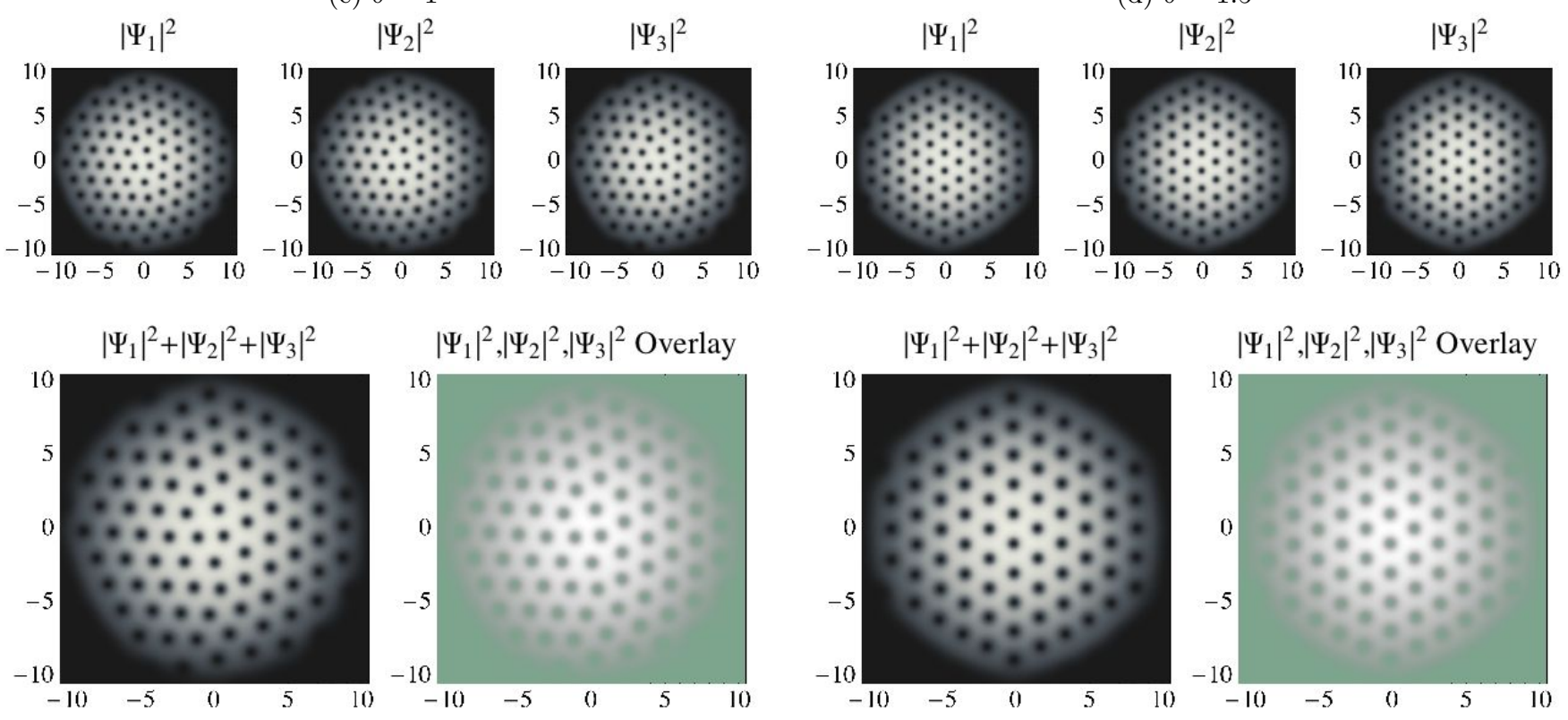

FIG. 1: (Color online) Some of the results obtained when the initial configuration used in the calculation is the same as the ground state of a non-rotating BEC. The top pictures in each panel are plots of the density profile for each component, $n_{i}=\left|\Psi_{i}\right|^{2}$. The bottom left picture is the full density profile $n=\left|\Psi_{1}\right|^{2}+\left|\Psi_{2}\right|^{2}+\left|\Psi_{3}\right|^{2}$, while the bottom right picture is the same plot, where the various components are distinguished by color (gray tones). Red regions correspond to zeros in the density of the first component, blue regions stay where the density of the second component vanishes, while green areas are the zeros of the third component density. However, no different colors (gray tones) are visible in this picture because vortices of all three components are coincident. The value of $\delta$ is reported on top of each panel. In all cases we obtain an integer vortex lattice.

expect that in a real situation the fluctuations of the trapped condensate will be responsible for the perturbation needed to eventually break the metastable integer vortices into the fractional ones. This behavior and the final structure of the three component lattice depend on the sign and on the magnitude of $g^{\prime}$, the coupling constant of the inter-component interactions. To characterize the system, let us define the ratio of the intercomponent and intra-component couplings by

$$
\delta \equiv g^{\prime} / g
$$

We examined a wide range of values for $\delta$, from 


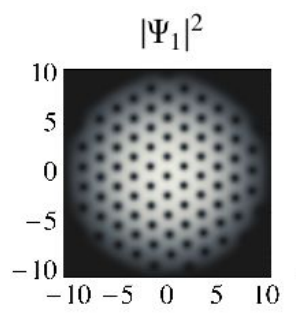

(a) $\delta=-0.1$
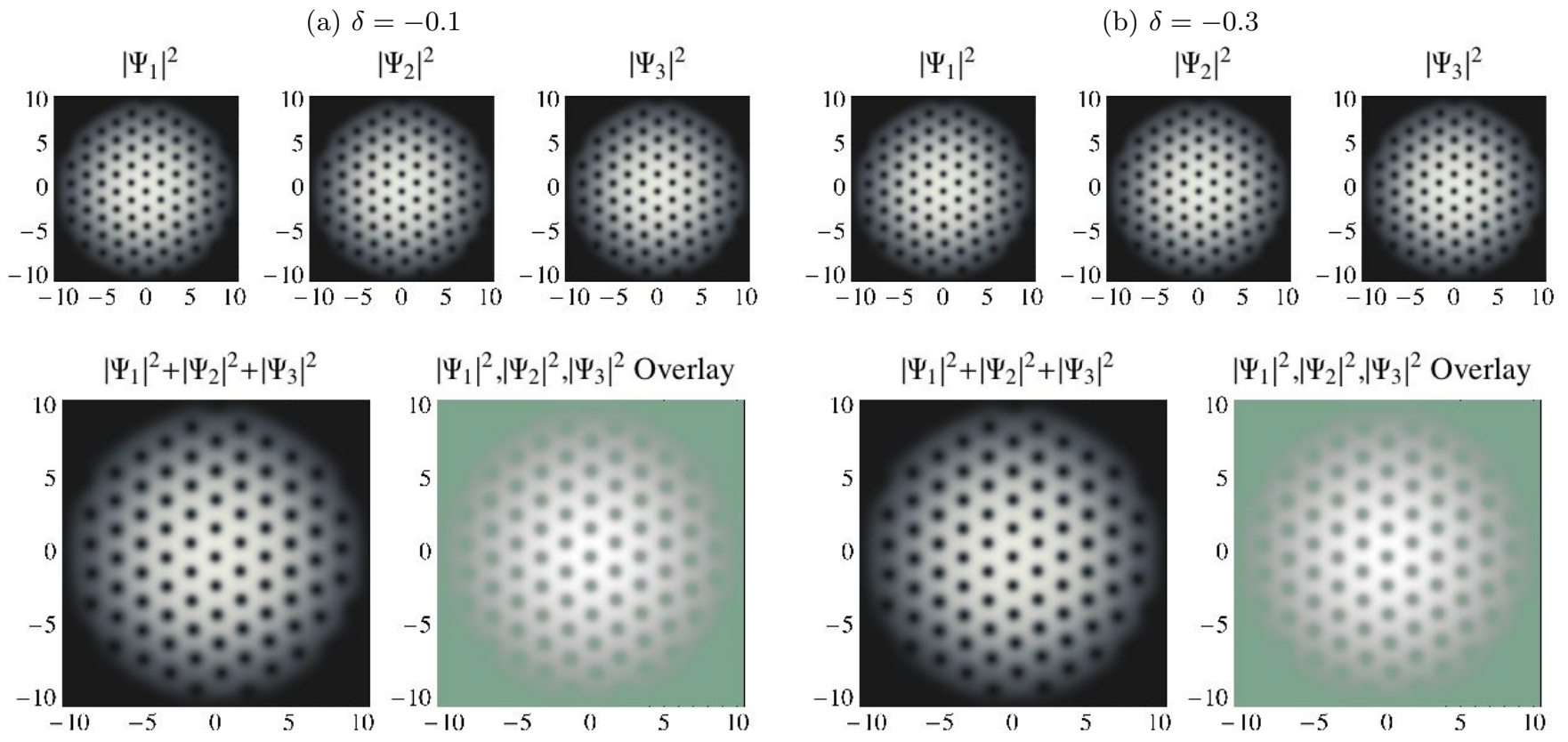

FIG. 2: (Color online) Integer vortex lattice obtained when $\delta<0$. The top pictures in each panel are plots of the single component density $n_{i}=\left|\Psi_{i}\right|^{2}$. The bottom left picture is the full density profile $n=\left|\Psi_{1}\right|^{2}+\left|\Psi_{2}\right|^{2}+\left|\Psi_{3}\right|^{2}$, while the bottom right picture is the same plot, where the various components are distinguished by color (gray tones). Red regions correspond to zeros in the density of the first component, blue regions stay where the density of the second component vanish, while green areas are the zeros of the third component density. However, no different colors (gray tones) are visible in this picture because vortices of all three components are coincident. Because of the attracting interaction, integer vortices do not split into fractional vortices.

$\delta_{\min }=-0.3$ to $\delta_{\max }=1.5$, with the separation $\Delta \delta=0.1$. In Fig. 2, we show the results we obtained for $\delta<0$. We obtained an Abrikosov lattice of integer vortices, as can be seen from the bottom pictures in each panel. When $\delta<0$, in fact, the interaction between the vortices belonging to different components is attractive [22] and, as expected, only integer vortices are formed and they arrange in an Abrikosov triangular lattice.

When $\delta=0$ each component is decoupled from the others and vortices of different components organize in an Abrikosov lattice independently.

The repulsive inter-component interaction present for $0<\delta<1$ [22] makes energetically convenient for the system to form fractional vortices instead of integer ones. The results obtained for this range of values for $\delta$ are reported in Figs. 3(a)-3(c). The schematic picture of the whole vortex lattice structure is drawn in Fig. 3(d). As can be seen from these figures, the fractional vortex lattice does not show any defect and both the total and individual lattices have the Abrikosov structure. This fact states the robustness of the shape of the fractional vortex lattice in a three-component BEC. One can find that vortices in each component constitute the Abrikosov lattice. Moreover, the repulsive force among vortices in different components determines relative positions of the Abrikosov lattice of each component, and consequently the total configuration is also in the form of the the Abrikosov lattice. This is possible because the number of components is three. In fact, in two-component BECs, there are two kinds of the vortex lattice structures depending on the inter-component coupling, that is, the Abrikosov's triangular lattice and square lattices. On the other hand, in our case of three component BEC, the Abrikosov lattice structure is robust in all ranges of the inter-component couplings for $0<\delta<1$.

If $\delta=1$, the symmetry of the system is enhanced to a $U(3)$ symmetry. A result is shown in Fig. 4(a). The situation is between the ordered Abrikosov lattice for $\delta<1$ and the vortex sheets for $\delta>1$. A new feature can be seen in this case: Density profiles of different components can have overlapped (almost) zeros. The right plot in the second row in Fig. 4 (a) shows a superposition of the density profiles of each component, distinguished by red, blue and green colors. However, other colors are present, which are generated by the mixing of the original ones: Yellow comes from the mixing of red and green, purple is the result of the mixing of red and blue, while cyan arises from the mixing of blue and yellow. In order to specify the positions of vortices, we can look at the plot of the phase of each of the order parameters, defined as $\phi_{i}=-i \ln \left(\Psi_{i} /\left|\Psi_{i}\right|\right)$. By using a schematic representation, reported in the last row of Fig. 4(a), we can compare the positions of the vortices in each component and can see that vortices are not coincident, even if the (almost) zeros of the density are overlapped. This happens because two vortices with different components are close to 


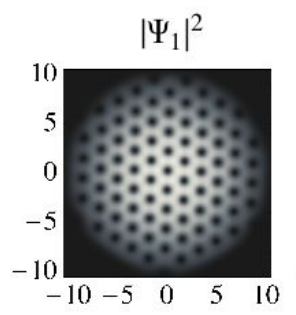

(a) $\delta=0.2$

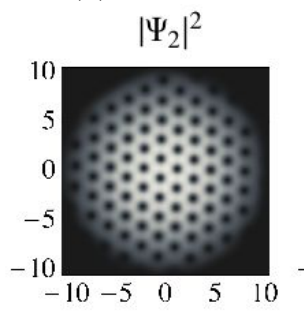

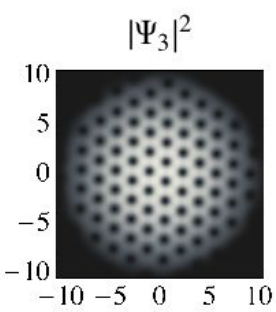

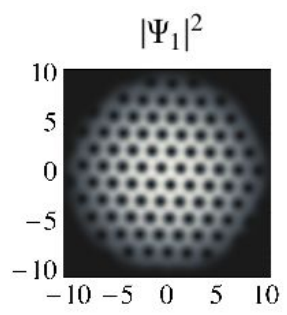

(b) $\delta=0.5$
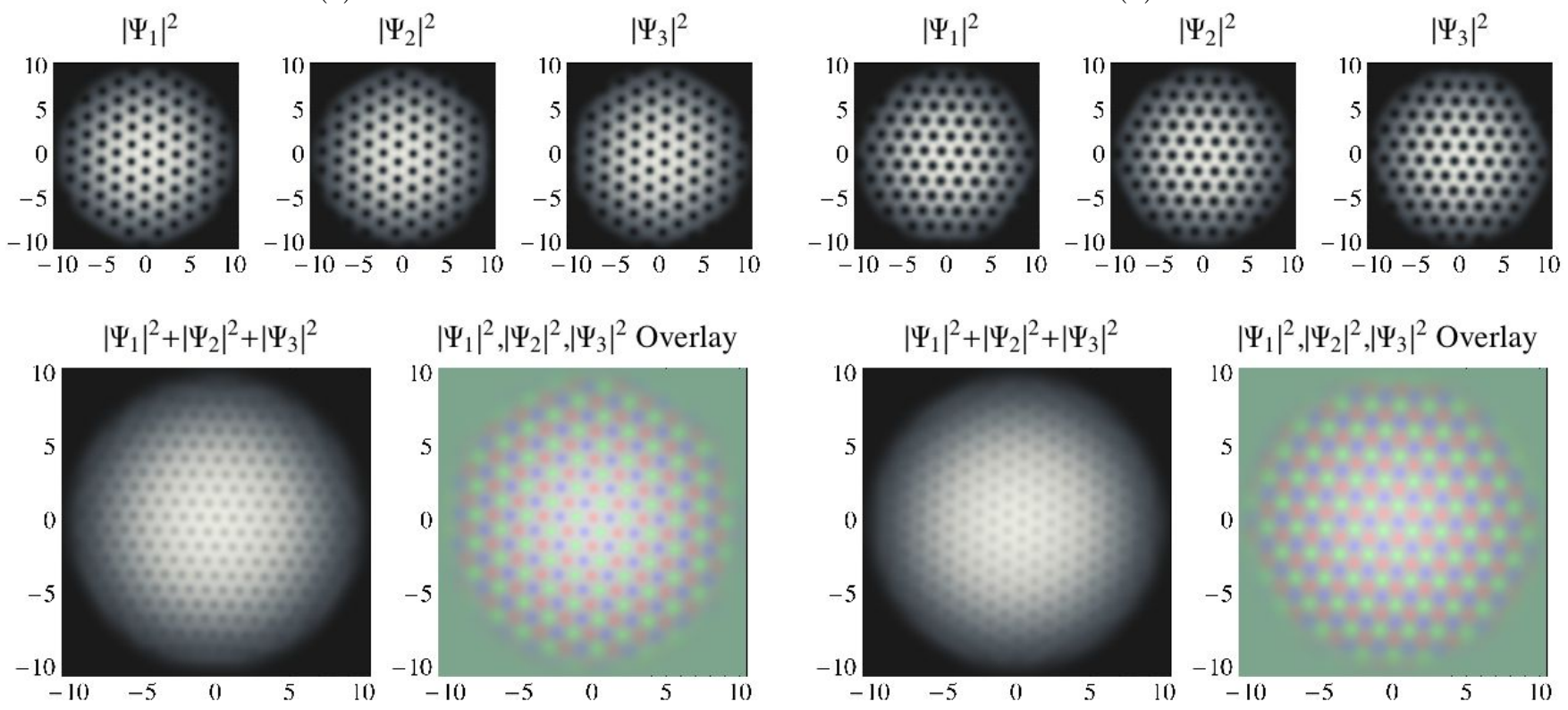

(c) $\delta=0.9$
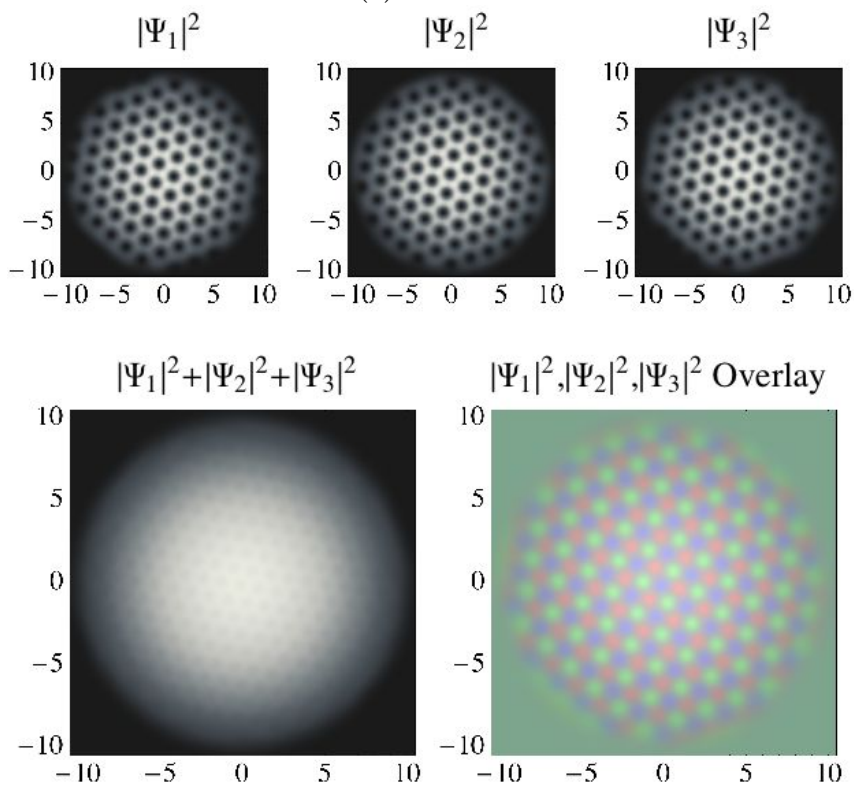

FIG. 3: (Color online) (a)-(c) One-third quantized vortex lattice for $g<g^{\prime}$. The top pictures in each panel are plots of the single component density $n_{i}=\left|\Psi_{i}\right|^{2}$. The bottom left picture is the full density profile $n=\left|\Psi_{1}\right|^{2}+\left|\Psi_{2}\right|^{2}+\left|\Psi_{3}\right|^{2}$, while the bottom right picture is the same plot, where the various components are distinguished by color (gray tones). Red regions correspond to zeros in the density of the first component, blue regions stay where the density of the second component vanish, while green areas are the zeros of the third component density. By this plot it can be seen that vortices in different components are well separated for this range of parameters. (d) A schematic picture of the $1 / 3$ quantized vortex lattice for $g<g^{\prime}$, where the different shapes and colors distinguish the different components.

each other in some place, although they never coincide. We also notice some symmetric structure in the lattice.

When $\delta>1$, the phase separation occurs and vortex sheets appear as in Fig. 4(b). This resembles the cases of two-component BECs, but new features arise. In this case, in a region where one component is present, the other two components are absent because of the phase separation so that zeros of the density profiles of the other two components are completely overlapped, as can be seen from the complete mixing of colors in the rightmost picture of the second row of Fig. 4 (b). By using the phase plot as done for the $\delta=1$ case, we observe that in a region where one component is nonzero and the amplitudes of the other two components are zero, there are 
(a) $\delta=1$
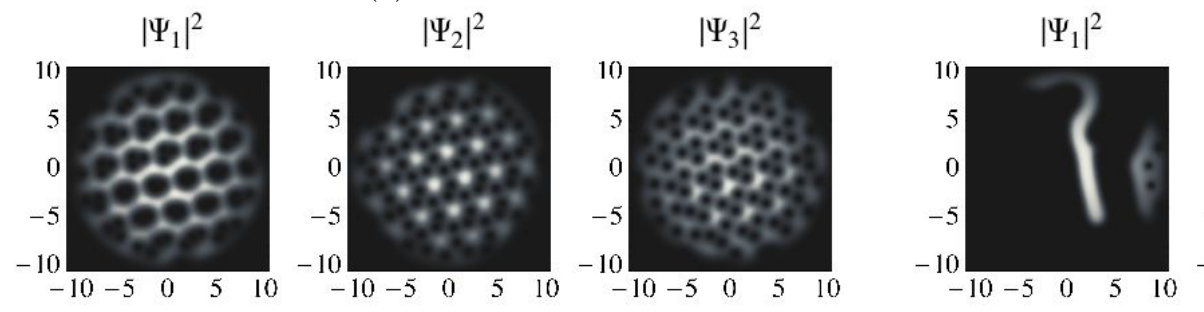

(b) $\delta=1.5$
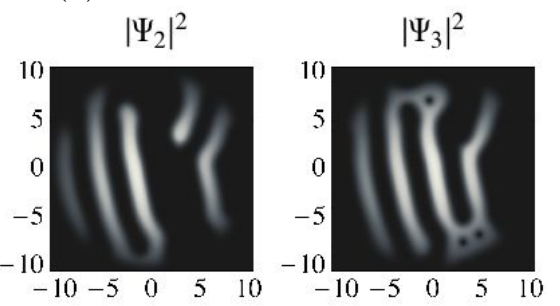
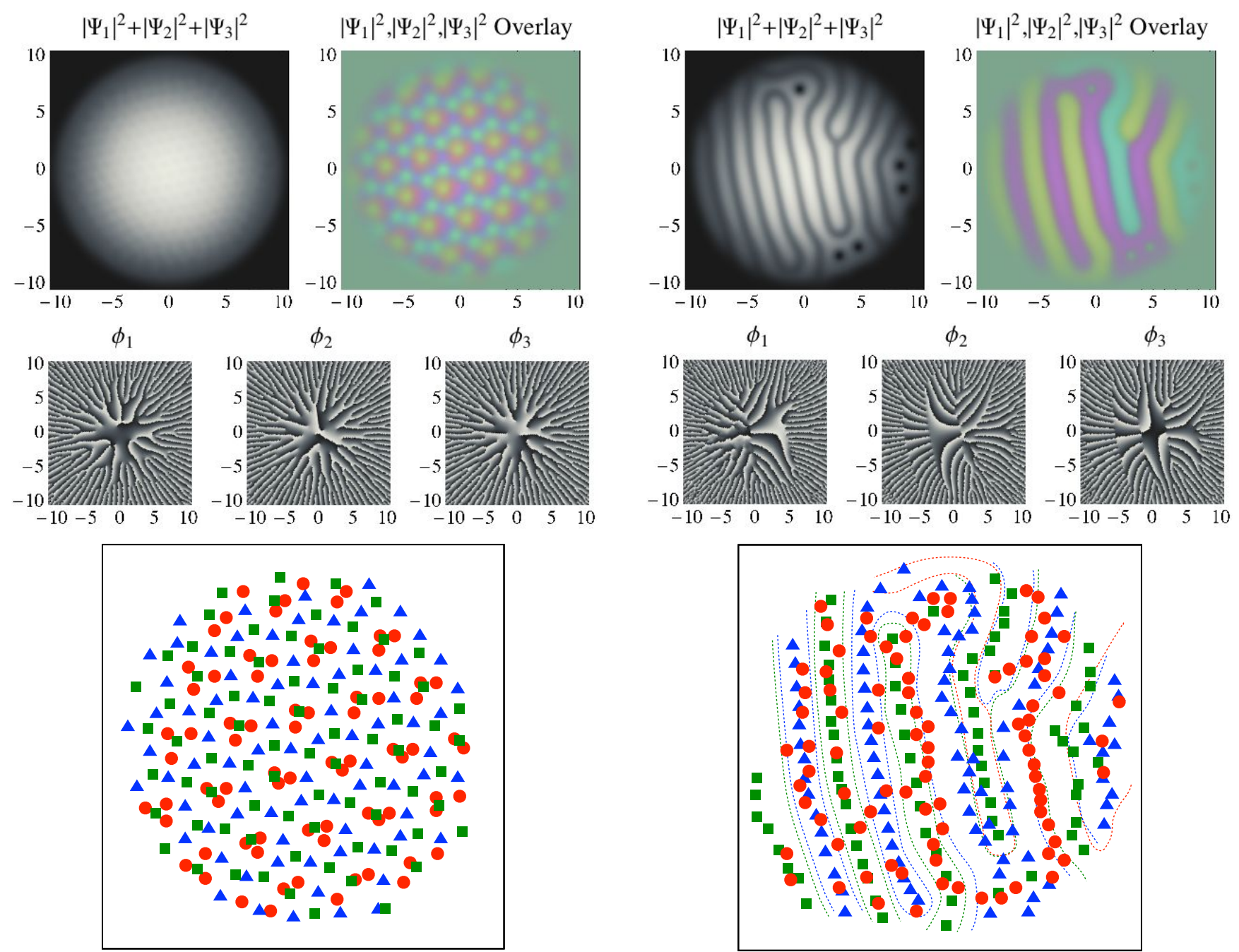

FIG. 4: (Color online) (a) One-third quantized vortex lattice for $g=g^{\prime}$ with the $U(3)$ symmetry, (b) vortex sheets for $g>g^{\prime}$. From the top in each column we show: plots of the single component density $n_{i}=\left|\Psi_{i}\right|^{2}$; plots of the full density profile $n=\left|\Psi_{1}\right|^{2}+\left|\Psi_{2}\right|^{2}+\left|\Psi_{3}\right|^{2}$, where in the rightmost picture the various components are distinguished by color (gray tones), with red corresponding to zeros in the density of the first component, blue staying where the density of the second component vanish, green corresponding to the zeros of the third component density; a plot of the phase of the order parameter of each component, $\phi_{i}=-i \ln \left(\Psi_{i} /\left|\Psi_{i}\right|\right)$ (black represents zero and white does $2 \pi$ ); a schematic drawing of the positions of vortices distinguished by colors and shapes, with the dashed lines in the right column being the domain walls separating sheets of different components. Because of the superposition of zeros of the density explained in the text, other colors appear, due to the mixing of the original colors: yellow, cyan and purple correspond to red-green, blue-green and red-blue mixing respectively.

ghost vortices of these two components whose positions are not coincident.

Moreover, this transition between an ordered lattice structure and the vortex sheets is quite sharp. In fact we report in Fig. 5 the results obtained for $\delta$ slightly greater than 1 , namely $\delta=1.05$. As can be seen form the plots, the vortices in the different components are not arranged in an ordered lattice, but they constitute vortex sheets. 

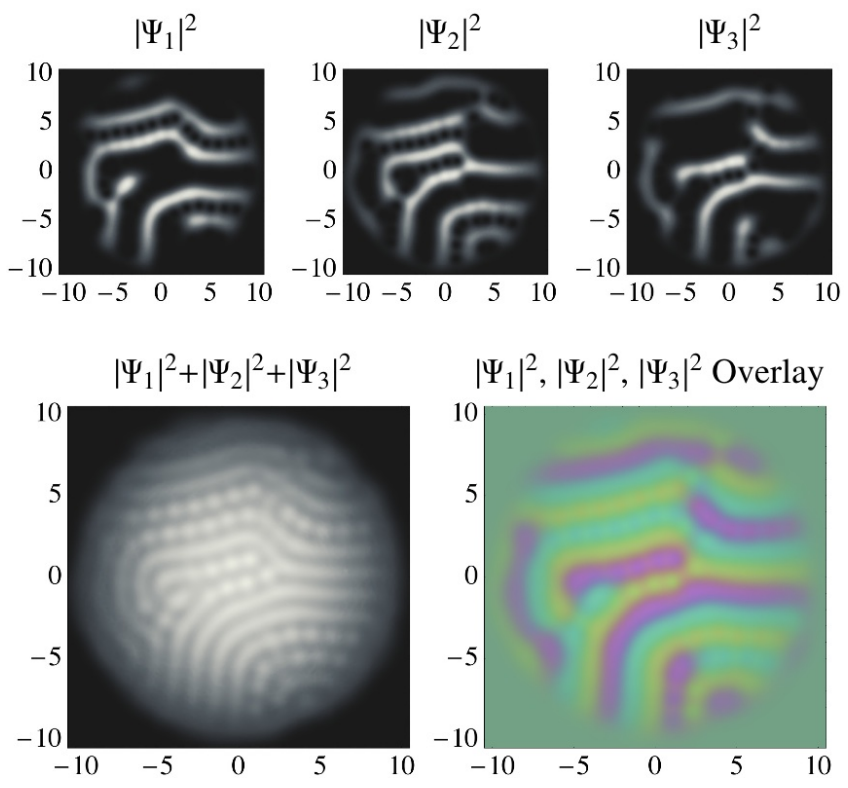

FIG. 5: (Color online) The results obtained for $\delta=1.05$. The top pictures are plots of the single component density $n_{i}=\left|\Psi_{i}\right|^{2}$. The bottom left picture is the full density profile $n=\left|\Psi_{1}\right|^{2}+\left|\Psi_{2}\right|^{2}+\left|\Psi_{3}\right|^{2}$, while the bottom right picture is the same plot, where the various components are distinguished by color (gray tones). Vortex sheets are formed for values of $\delta$ just above 1 .

\section{SUMMARY AND DISCUSSION}

In conclusion we have found a robustness of the ordered Abrikosov's triangular lattices of fractional vortices in three-component BECs in a large parameter region, where the inter-component coupling is less than the intracomponent coupling $g^{\prime}<g$. Our finding is in contrast to the cases of two-component BECs, where triangular and square lattice are present depending on the intercomponent coupling constant and the rotation speed. In the range $g \leq g^{\prime}(\delta<1)$, we find that (almost) zeros of the density of different components can coincide, even if the vortices lying inside these regions are separated. When $g=g^{\prime}(\delta=1)$, two vortices in different components are close in some places, where we see the overlap of almost zeros of these two components. When $g<g^{\prime}$ $(\delta>1)$, vortex sheets appear similarly to the case of two component BECs. In a region where one component is present, the other two components must vanish because of the phase separation, and in that region there are ghost vortices in these two components whose positions do not coincide.

Our results imply that in the region $0<\delta<1$ triangular structures of colorful vortex lattices in three components are very robust, in contrast to the cases of two components. This happens because the number of components, three, coincides with the number of vertices of a triangle. The implication to a colorful vortex lattice in the color superconductor is suggestive. Since both the number of color and the flavors (up, down, strange) are three, it implies a very robust colorful vortex lattice.

Because of our motivation to simulate a color superconductor, we restrict to the case of the symmetric couplings in Eq. 22. Apart from this motivation, we could change couplings asymmetrically. If we change either $g_{i}, g_{i j}, m_{i}$ or $\mu_{i}$, circulations of vortices are not $1 / 3$ quantized anymore. When we consider multi-component BECs as a mixture of different atoms, this happens in general. The vortex phase diagram in such a case has been studied recently for two-component BECs [18]. These remain as a future problem.

If we set $g_{23}=g_{31}=0$, the third component $\Psi_{3}$ decouples from the others, reducing to two-component BECs of $\Psi_{1}$ and $\Psi_{2}$. There, we have the phase diagram of the two component BECs [13 19]. It is an interesting future problem how the vortex phase of two-component BECs is connected to that of a three-component $\mathrm{BEC}$ found in this paper by gradually increasing the coupling to $\Psi_{3}$.

Another interesting problem is to introduce internal coherent couplings between different components via Rabi oscillations. In the case of two-component BECs, two different fractional vortices are combined by a sineGordon domain wall to become a two-vortex molecule or a dimer [20]. The effects of this term in the vortex phase diagram have been studied recently by the present authors [19]. For three-components, the introduction of internal coherent couplings results in a three-vortex molecule, a trimer [23], and the same for $N$-component results in a vortex $N$-omer 24 .

In color superconductors of high density quark matter, modulated phases, called as crystalline color superconductors, are proposed 43 . Our configurations of vortex sheets at $\delta>1$ consist of domain walls, their junctions and vortices absorbed into them. These configurations may be useful to understand how vortices are trapped in the modulations in crystalline color superconductors.

Before closing the paper, let us make a comment on a possible realization in experiments. Two-component BECs of different hyperfine states of the same atom have been already realized using the $|1,-1\rangle$ and $|2,1\rangle$ states [44] and the $|2,1\rangle$ and $|2,2\rangle$ states [45] of ${ }^{87} \mathrm{Rb}$, respectively. Systems with three components can be realized in principle by using a mixture of the above mentioned states of ${ }^{87} \mathrm{Rb}$ via an optical trap [46, and our prediction is testable in laboratory experiments.

\section{Acknowledgements}

M. C. thanks the Department of Physics at Hiyoshi, Keio University, for warm hospitality in the beginning of this project. M. N. thanks INFN, Pisa, for partial support and hospitality while this work was done. Both authors thank Walter Vinci and Kenichi Konishi for useful discussions. The numerical calculations were performed on the INFN CSN4 cluster located in Pisa. The work of M. N. is supported in part by a Grant-in-Aid for Sci- 
entific Research (No. 23740198 and 25400268) and by the "Topological Quantum Phenomena" Grant-in-Aid for Scientific Research on Innovative Areas (No. 23103515 and 25103720) from the Ministry of Education, Culture, Sports, Science and Technology (MEXT) of Japan.
[1] M. M. Salomaa and G. E. Volovik, Phys. Rev. Lett. 55, 1184-1187 (1985); M. M. Salomaa and G. E. Volovik, Rev. Mod. Phys. 59, 533-613 (1987).

[2] G. E. Volovik, The Universe in a Helium Droplet, Clarendon Press, Oxford (2003).

[3] D. A. Ivanov, Phys. Rev. Lett. 86, 268 (2001).

[4] S. B. Chung, H. Bluhm, and E.-A. Kim, Phys. Rev. Lett. 99, 197002 (2007); S. B. Chung and S. A. Kivelson, Phys. Rev. B 82, 214512 (2010).

[5] J. Jang, D. G. Ferguson, V. Vakaryuk, R. Budakian, S. B. Chung, P. M. Goldbart and Y. Maeno, Science 331, 6014, 186 (2011).

[6] Y. Tanaka, J. Phys. Soc. Jp. 70 (2001) 2844; Phys. Rev. Lett. 88 (2001) 017002.

[7] E. Babaev, Phys. Rev. Lett. 89 (2002) 067001; E. Babaev, A. Sudbo and N. W. Ashcroft, Nature 431, 666 (2004); J. Smiseth, E. Smorgrav, E. Babaev and A. Sudbo, Phys. Rev. B 71, 214509 (2005); E. Babaev and N. W. Ashcroft, Nature Phys. 3, 530 (2007).

[8] A. Gurevich and V. M. Vinokur, Phys. Rev. Lett. 90, 047004 (2003).

[9] J. Goryo, S. Soma and H. Matsukawa, Euro Phys. Lett. 80, 17002 (2007).

[10] T.-L. Ho, Phys. Rev. Lett. 81, 742-745 (1998); T. Ohmi and K. Machida, J. Phys. Soc. Jpn. 67, 1822 (1998).

[11] G. W. Semenoff and F. Zhou, Phys. Rev. Lett. 98 (2007) 100401; M. Kobayashi, Y. Kawaguchi, M. Nitta and M. Ueda, Phys. Rev. Lett. 103 (2009) 115301.

[12] D. T. Son and M. A. Stephanov, Phys. Rev. A 65, 063621 (2002).

[13] E. J. Mueller and T.-L. Ho, Phys. Rev. Lett. 88, 180403 (2002).

[14] K. Kasamatsu, M. Tsubota and M. Ueda, Phys. Rev. Lett. 91, 150406 (2003).

[15] K. Kasamatsu, M. Tsubota and M. Ueda, Int. J. Mod. Phys. B 19, 1835 (2005).

[16] S. J. Woo, Q-Han Park, and N. P. Bigelow, Phys. Rev. Lett. 100, 120403 (2008).

[17] A. Aftalion, P. Mason and W. Juncheng, Phys. Rev. A 85, 033614 (2012).

[18] P. Kuopanportti, J. A. M. Huhtamäki, M. Möttönen, Phys. Rev. A 85, 043613 (2012).

[19] M. Cipriani, and M. Nitta, "Crossover between integer and fractional vortex lattices in coherently coupled twocomponent Bose-Einstein condensates," arXiv:1303.2592 [cond-mat.quant-gas].

[20] K. Kasamatsu, M. Tsubota and M. Ueda, Phys. Rev. Lett 93, 250406 (2004).

[21] K. Kasamatsu, and M. Tsubota, Phys. Rev. A 79, 023606 (2009).

[22] M. Eto, K. Kasamatsu, M. Nitta, H. Takeuchi and M. Tsubota, Phys. Rev. A 83, 063603 (2011).

[23] M. Eto and M. Nitta, Phys. Rev. A 85, 053645 (2012).

[24] M. Eto and M. Nitta, "Vortex graphs as $N$-omers and C $P^{N-1}$ Skyrmions in $N$-component Bose-Einstein condensates," arXiv:1303.6048 [cond-mat.quant-gas].
[25] Y. G. Rubo, Phys. Rev. Lett. 99, 106401 (2007); K. G. Lagoudakis, T. Ostatnicky, A. V. Kavokin, Y. G. Rubo, R. Andre, and B. Deveaud-Pledran, Science 326, 974 - 976 (2009); Y. G. Rubo, arXiv:1209.6538 [cond-mat.mes-hall] G. Roumpos, M. D. Fraser, A. Löffler, S. Höfling, A. Forchel and Y. Yamamoto Nature Phys. 7, 129-133 (2011).

[26] J. Keeling and N. G. Berloff, Phys. Rev. Lett. 100, 250401 (2008); M. O. Borgh, G. Franchetti, J. Keeling and N. G. Berloff, Phys. Rev. B 86, 035307 (2012).

[27] L. M. Pismen, Phys. Rev. Lett. 72, 2557-2560 (1994); L. M. Pismen, Physica D 73, 244-258 (1994); I. S. Aranson and L. M. Pismen, Phys. Rev. Lett. 84, 634-637 (2000); L. M. Pismen, Vortices in Nonlinear Fields: From Liquid Crystals to Superfluids, from Non-Equilibrium Patterns to Cosmic Strings, Oxford Univ Pr on Demand (1999).

[28] A. P. Balachandran, S. Digal and T. Matsuura, Phys. Rev. D 73 (2006) 074009.

[29] E. Nakano, M. Nitta, T. Matsuura and, Phys. Rev. D 78, 045002 (2008); Prog. Theor. Phys. Suppl. 174, 254 (2008).

[30] M. Eto and M. Nitta, Phys. Rev. D 80 (2009) 125007.

[31] M. Eto, E. Nakano and M. Nitta, Phys. Rev. D 80, 125011 (2009); M. Eto, M. Nitta and N. Yamamoto, Phys. Rev. Lett. 104, 161601 (2010); M. Eto, M. Nitta, N. Yamamoto and , Phys. Rev. D 83, 085005 (2011); Y. Hirono, T. Kanazawa, and M. Nitta, Phys. Rev. D 83, 085018 (2011); A. Gorsky, M. Shifman and A. Yung, Phys. Rev. D 83, 085027 (2011).

[32] Y. Hirono, and M. Nitta, Phys. Rev. Lett. 109, 062501 (2012).

[33] W. Vinci, M. Cipriani, M. Nitta and, Phys. Rev. D 86, 085018 (2012).

[34] M. Cipriani, W. Vinci, M. Nitta and, Phys. Rev. D 86, 121704 (2012).

[35] C. J. Pethick and H. Smith, Bose-Einstein Condensation in Dilute Gases, 2nd ed. (Cambridge University Press, Cambridge 2008)

[36] C. Chin, R. Grimm, P. Julienne and E. Tiesinga, Rev. Mod. Phys. 82, 1225 (2010).

[37] C. J. Myatt, E. A. Burt, R. W. Ghrist, E. A. Cornell, and C. E. Wieman, Phys. Rev. Lett. 78, 586 (1997); D. S. Hall, M. R. Matthews, J. R. Ensher, C. E. Wieman, and E. A. Cornell, Phys. Rev. Lett. 81, 1539 (1998); M. Mertes, J. W. Merrill, R. Carretero-González, D. J. Frantzeskakis, P. G. Kevrekidis, and D. S. Hall, Phys. Rev. Lett. 99, 190402 (2007); S. Tojo, Y. Taguchi, Y. Masuyama, T. Hayashi, H. Saito, and T. Hirano, Phys. Rev. A 82, 033609 (2010).

[38] G. Modugno, M. Modugno, F. Riboli, G. Roati, and M. Inguscio, Phys. Rev. Lett. 89, 190404 (2002); G. Thalhammer, G. Barontini, L. De Sarlo, J. Catani, F. Minardi, and M. Inguscio, Phys. Rev. Lett. 100, 210402 (2008).

[39] S. B. Papp, J. M. Pino, and C. E. Wieman, Phys. Rev. 
Lett. 101, 040402 (2008).

[40] D. J. McCarron, H. W. Cho, D. L. Jenkin, M. P. Köppinger, and S. L. Cornish, Phys. Rev. A 84, 011603(R) (2011).

[41] M. G. Alford, K. Rajagopal and F. Wilczek, Nucl. Phys. B 537, 443 (1999); K. Rajagopal and F. Wilczek, In *Shifman, M. (ed.): At the frontier of particle physics, vol. $3^{*}$ 2061-2151 hep-ph/0011333; M. G. Alford, A. Schmitt, K. Rajagopal and T. Schafer, Rev. Mod. Phys. 80, 1455 (2008).

[42] T. Mizushima, K. Machida, and T. Kita, Phys. Rev. Lett. 89, 030401 (2002); T. Mizushima, K. Machida, and T. Kita, Phys. Rev. A 66, 053610 (2002); T. Kita, T. Mizushima, and K. Machida, Phys. Rev. A 66,
061601(R) (2002); T. Mizushima, N. Kobayashi, and K. Machida, Phys. Rev. A 70, 043613 (2004).

[43] R. Anglani, R. Casalbuoni, M. Ciminale, R. Gatto, N. Ippolito, M. Mannarelli and M. Ruggieri, arXiv:1302.4264 [hep-ph].

[44] M. R. Matthews, B. P. Anderson, P. C. Haljan, D. S. Hall, C. E. Wieman, and E. A. Cornell, Phys. Rev. Lett. 83, 2498 (1999).

[45] P. Maddaloni, M. Modugno, C. Fort, F. Minardi, and M. Inguscio, Phys. Rev. Lett. 85, 2413 (2000).

[46] C. Hamner, J. J. Chang, P. Engels, and M. A. Hoefer, Phys. Rev. Lett. 106, 065302 (2011). 